\title{
Radially polarized, half-cycle, attosecond pulses from laser wakefields through coherent synchrotron-like radiation
}

\author{
F. Y. $\mathrm{Li}^{1}$, Z. M. Sheng ${ }^{1,2},{ }^{*}$ M. Chen ${ }^{1},{ }^{\dagger}$ L. L. Yu ${ }^{1}$, J. Meyer-ter-Vehn ${ }^{3}$, W. B. Mori ${ }^{4}$, and J. Zhang ${ }^{1}$ \\ ${ }^{1}$ Key Laboratory for Laser Plasmas (Ministry of Education) and Department of Physics and Astronomy, \\ Shanghai Jiao Tong University, Shanghai 200240, China \\ ${ }^{2}$ SUPA, Department of Physics, University of Strathclyde, Glasgow G4 ONG, UK \\ ${ }^{3}$ Max-Planck-Institut für Quantenoptik, D-85748 Garching, Germany \\ ${ }^{4}$ University of California, Los Angeles, California 90095-1547, USA
}

\begin{abstract}
Attosecond bursts of coherent synchrotron-like radiation are found when driving ultrathin relativistic electron disks in a quasi-one-dimensional regime of wakefield acceleration, in which the laser waist is larger than the wake wavelength. The disks of overcritical density shrink radially due to focusing wake fields, thus providing the transverse currents for the emission of an intense, radially polarized, half-cycle pulse of about 100 attoseconds in duration. The electromagnetic pulse first focuses to a peak intensity $\left(7 \times 10^{20} \mathrm{~W} / \mathrm{cm}^{2}\right) 10$ times larger than the driving pulse and then emerges as a conical beam. Basic dynamics of the radiative process are derived analytically and in agreement with particle-in-cell simulations. By making use of gas targets instead of solids to form the ultrathin disks, the new scheme allows for high repetition rates required for applications.
\end{abstract}

PACS numbers: 52.38.Kd, 52.59.-f, 52.59.Ye, 42.65.Ky

\section{Introduction}

Great efforts have recently been devoted to obtain attosecond bursts of coherent extreme-ultraviolet (XUV) and soft $\mathrm{X}$-ray radiations. They open the door to exploring and controlling processes of the microcosm such as attosecond spectroscopy of bound electrons [1]. So far, established methods for producing such pulses are based on high harmonic generation from atomic gases, where the driving laser intensity is limited below $10^{16} \mathrm{~W} / \mathrm{cm}^{2}$ [2]. High harmonic generation from solid plasma surface [3] makes use of relativistic optics ( $>10^{18} \mathrm{~W} / \mathrm{cm}^{2}$ ) [4] and allows for much brighter sources. These harmonics correspond to trains of attosecond pulses in the time domain, and can be used to isolate single attosecond spikes using high-pass filters [5]. By temporally rotating either the surface geometry [6] or the driver wavefront [7], individual spikes can be obtained even without filtering. Single attosecond pulses can also be produced from relativistic electron sheets formed by laser interaction with nanometer foils [8] or by nano-bunching at solid surfaces [9]. Wu et al. considered a double-foil setup irradiated by an ultraintense few-cycle laser pulse and the generation of a relativistic electron sheet from the first production foil by direct laser acceleration. Under oblique incidence, laser beam reflection at the second foil provides the transverse current, which emits an intense $p$-polarized half-cycle attosecond pulse [10].

In the present paper, we propose a new path, producing single intense attosecond pulses from underdense plasmas rather than solids. By making use of gas plasmas as a target, the condition of ultrahigh laser contrast is greatly relaxed and a bright source allowing for high repetition rates may become possible. The new scheme is based on plasma wave acceleration driven by an intense short laser pulse. It has been known

\footnotetext{
*zmsheng@sjtu.edu.cn

or zhengming.sheng@strath.ac.uk

†minchen@sjtu.edu.cn
}

that these relativistic wake waves exhibit strong accelerating and focusing fields for electrons, and promise acceleration to high energies over a short distance [11]. In most experiments the laser beam is tightly focused to reach highest possible intensity $I_{0}$ with waist $W_{0} \leq \sqrt{a_{0}} \lambda_{p} / \pi$, where $\lambda_{p}$ is the plasma wavelength and $a_{0}=8.5 \times 10^{-10} \lambda_{0}[\mu \mathrm{m}] \sqrt{\mathrm{I}_{0}\left[\mathrm{~W} / \mathrm{cm}^{2}\right]}$ the normalized laser amplitude at wavelength $\lambda_{0}$. Plasma electrons within the laser path is then pushed sideways by strong laser radiation pressure in the transverse direction $\left(\propto \nabla_{r} I\right)$, creating bubble-like wakes. Some electrons circling around the bubble sheath are transversely injected at the rear vertex [12] and accelerated forming a narrow bunch [13]. This is the concept of bubble wakefield acceleration [14]. An important feature relevant here is that the accelerating bunch oscillates in the wake wave forced by the focusing fields and emits bright betatron X-rays [15].

Here, we make use of a different regime of wakefield acceleration, occurring for driving laser waist larger than the wake wavelength. We refer to it as quasi-one-dimensional (quasi1D) regime, since wake electrons are now dominated in 1D longitudinal motion, at least for those close to laser axis. Correspondingly, density crests of the plasma wave show disklike profile as visualized in experiments [16]. Most notably, they converge into dense electron sheets when the wake is driven to high nonlinearities or close to wave breaking. These ultrathin density crests have been used as relativistic flying mirrors for laser backscattering [17]. Attosecond XUV/Xray pulses can be produced in this way due to time compression and frequency up-conversion by Doppler shift factors of $\sim 4 \gamma_{p}^{2}$, where $\gamma_{p}=\left(1-v_{p}^{2} / c^{2}\right)^{-1 / 2}$ with $v_{p}$ the phase velocity of the plasma wave.

In the present work, we follow another line that does not require a second pulse, but leads to self-emission of an intense attosecond pulse from the dense electron sheet itself. For this to happen, we consider breaking of the above wake-wave symmetries that leads to injection of an ultrathin electron disk (or electron sheet) into the wakefield. As we shall see, the electron sheet contracts in transverse direction right after injection 
due to focusing wake fields, while boosted in energy primarily by longitudinal wakefield acceleration. The central new observation here is that a strong radially polarized, half-cycle, attosecond (RHA) pulse is emitted by the transverse currents established during the contraction phase. This coherent attosecond burst of electromagetic radiation differs in several aspects from the wakefield based betatron x-rays normally obtained so far [15]. It is also found that wakefield acceleration plays a crucial role in amplifying the radiated fields. Radiation reaction damps and eventually saturates the fields, resulting in a high-energy (few millijoule) attosecond pulse of relativistic intensity.

The remainder of the paper is organized as follows. Section II discusses how to inject an electron sheet into a wakefield and, in particular, a self-injection scenario based on density transition is briefly summarized. In Sec. III, the generation of RHA pulses from an injected electron sheet is described using particle-in-cell (PIC) simulations. Comparisons with the betatron radiations normally obtained from bubble acceleration are discussed. In Sec. IV, a simplified model is built for the illustration of the basic dynamics of RHA generation. In Sec. V, additional multi-dimensional effects beyond the simplified model are discussed and verified using PIC simulations. Scalings of RHA generation with different laser parameters are also studied. Finally, section VI summarizes the whole paper and discusses the experimental conditions required.

\section{Electron sheet injection into quasi-1D wakefield}

Different ways may, in principle, be used to induce injection of electron sheets into a wakefield [18]. A direct and promising approach was recently described using density-gradient injection in a controlled manner. The details of the scheme are documented in Ref. [19], where an up-ramp density profile followed by a plateau is employed. Wave breaking then occurs sharply at the density transition position, leading to a sudden longitudinal injection into the quasi-1D wakefield. This is in contrary to the transverse injection that normally occurs quasi-continuously in the bubble regime $[12,14]$. Notice that longitudinal injection but in a gentle way has been observed in experiments [20]. The key elements of the present sharp longitudinal injection are summarized as follows. First, along the up-ramp, the first few wake wave periods trailing after the driving laser can travel at superluminal phase speeds for high enough density gradients. It is also valid for high wave nonlinearities, so that the density wave crests can be stably compressed into dense electron sheets without premature injection $[19,21]$. The free of injection eventually terminates as the wake wave propagates into the following density plateau region, where the wave's phase velocity falls below the light speed. As a result, a major part of the sheet electrons satisfying the injection threshold are trapped as whole in a very short time scale. They typically form an ultrathin (10s nanometers thick corresponding to attoseconds in duration) overcritical dense electron disk that accelerates in the wakefield.

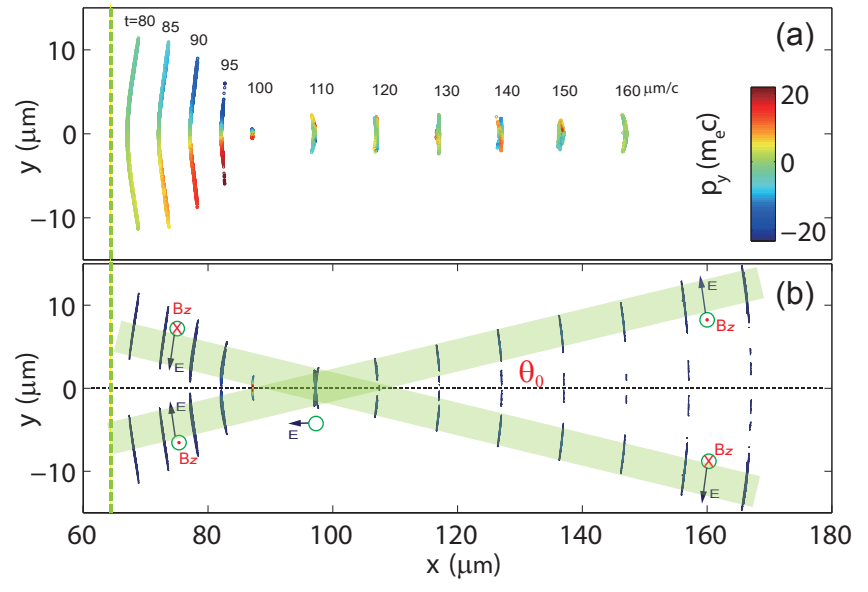

FIG. 1: (Color online). (a) Snapshots of a trapped electron sheet at different times (given as label) colored according to transverse momenta $p_{y}$ and (b) the radiated attosecond pulses given as contour plots of $B_{z}^{2}$. The dashed line on the left side marks the position of sharp injection of the electron sheet. The full density pattern of the wake wave is given in Fig. 2(b) for $t=160 \mu \mathrm{m} / \mathrm{c}$. These results are obtained from 2D PIC simulations in $x-y$ plane. In full 3D geometry, the emission appears as an annular beam propagating as a radially polarized, half-cycle, attosecond (RHA) pulse along a cone with opening angle $\theta_{0} \sim 10^{\circ}$, indicated as shaded stripes.

\section{Attosecond pulse generation via particle-in-cell simulation}

The evolution of an injected electron sheet in a wakefield and the associated radiation flash are first illustrated in Figs. 1(a) and 1(b). It is seen that the sheet contracts in transverse direction to a smallest diameter in tens of laser periods right after the injection, while accelerating longitudinally up to highly relativistic $\gamma$ factors (compare with Fig. 4). It is in this short time interval that the attosecond pulse is emitted. As found in Fig. 1(b), the pulse is first attached to the electron sheet, but then propagates along a cone, while the electrons move closer to the axis in a channel of almost constant diameter.

These results are obtained from 2D PIC simulations using the code OSIRIS [22]. As a demonstration case, the controlled self-injection scenario [19] is employed to induce the sheet injection. The simulation parameters are detailed as follows. A linearly polarized (along $z$ direction), 18 fs (FWHM of intensity) laser pulse of peak intensity $I_{0}=7.7 \times 10^{19} \mathrm{~W} / \mathrm{cm}^{2}$ irradiates an underdense plasma slab with a density ramping-up front of $65 \mu \mathrm{m}$ long. The plateau density is $n_{0}=0.04 n_{c}$ with $n_{c}=1.74 \times 10^{21} \mathrm{~cm}^{-3}$ the critical density for laser wavelength $\lambda_{0}=0.8 \mu \mathrm{m}$. A laser waist of $W_{0}=17 \mu \mathrm{m}$ is used and large enough for the formation of wide electron sheets in the quasi-1D regime. An electron temperature of $T_{e}=20 \mathrm{eV}$ is initialized to mimic field ionization by prepulses. In the simulation, spatial grids of $\Delta x \times \Delta y=158 \times 25$ per square micron and time steps of $\Delta t=0.00625 \mu \mathrm{m} / \mathrm{c}$ are chosen and fine enough to resolve the attosecond features; we have checked that even higher resolutions give nearly the same results. 
We now analyze the radiative process in general. The injected electrons are diverted by focusing wake fields toward the central axis according to $d \beta_{\perp} / d t \simeq \varepsilon_{\perp} / \gamma$. Here, $\beta_{\perp}$ is the normalized transverse velocity, and time $t$ and focusing fields $\varepsilon_{\perp}$ are, respectively, normalized by $\omega_{0}^{-1}$ and $E_{0}=m_{e} \omega_{0} c / e$ with electron mass $m_{e}$, elementary charge $e$ and laser frequency $\omega_{0}=2 \pi c / \lambda_{0}$. Near the center axis, the focusing field, $\varepsilon_{\perp} \propto n_{0} r$, is almost linear in both radius $r$ and ambient density $n_{0}$. For the present conditions, the betatron strength parameter [23] of trapped electrons $K=\gamma k_{\beta} r_{\beta}$ typically amounts to a few tens, where $k_{\beta}=2 \pi / \lambda_{\beta}$ with $\lambda_{\beta}=(2 \gamma)^{1 / 2} \lambda_{p}$ the betatron wavelength and $r_{\beta}$ is the amplitude of the betatron orbit. Thus these electrons perform synchrotron-like motion with curvature radius $\rho \simeq \lambda_{0} \gamma /\left(2 \pi \varepsilon_{\perp}\right)$ and emit broad-band radiation with cut-off frequency $\omega_{c} \simeq 3 \gamma^{3} c \rho^{-1}$ [24]. There are a couple of new features compared with normal betatron radiations [15]. First, the RHA pulses are produced at the initial contraction phase, corresponding to subcycle (or more accurately, a quarter of cycle) of betatron oscillation, during which the radiation generation can be modeled with relativistic electron sheets. Second, the high density of the electron sheet, typically larger than the critical density $\sim 10^{21} \mathrm{~cm}^{-3}$ [19], enables synchrotron-like radiation in a coherent manner. Coherency occurs provided that sufficiently large number of electrons reside in a volume of the radiation wavelength dimension in the rest frame of electrons. For the present case of self-emission, it requires the sheet density to satisfy

$$
n_{s} \gg 10^{13} \varepsilon_{\perp}^{3} \gamma^{4} \mathrm{~cm}^{-3},
$$

where radiation at the cutoff wavelength $\lambda_{c}=2 \pi c / \omega_{c}$ is assumed. Considering that $\varepsilon_{\perp} \sim 0.1$, this criterion can be readily met even for $\gamma \sim 100$. As a result, the coherency is well preserved at the initial contraction phase, during which the trapped sheet electrons are only accelerated up moderate energies (less than $50 \mathrm{MeV}$ in the present case). After that the sheet electrons are confined within a small radius and shall emit normal betatron X-rays which are incoherent and orders of magnitude weaker in intensity. Third, due to axial symmetry and inward acceleration of disk electrons, radially polarized half-cycle electromagnetic pulses are produced during the contraction. Here, owing to the ultrarelativistic feature of sheet electrons, the dephasing time arising from velocity mismatch between the coherent radiation and the electron sheet is much longer than the contraction period (i.e., from injection to focused to the laser axis), which is typically a few tens of laser periods as shown in Fig. 1(a). Therefore, the radiated pulse is almost overlapped with the sheet profile during the contraction phase and naturally has an attosecond duration. It is interesting to mention that radially polarized half-cycle pulses have also been recently demonstrated from thin photoconductors, where a sequence of annulus microelectrodes are fabricated on a wafer surface to induce radial currents [25]. However, due to the static wafer plane, only pulses of picosecond duration are generated, determined by the lifetime of the radiating currents.

These features are well illustrated by the sample simulation. Figure 2(a) records how the peak field $B_{z}^{\max } / B_{0}$ of the RHA
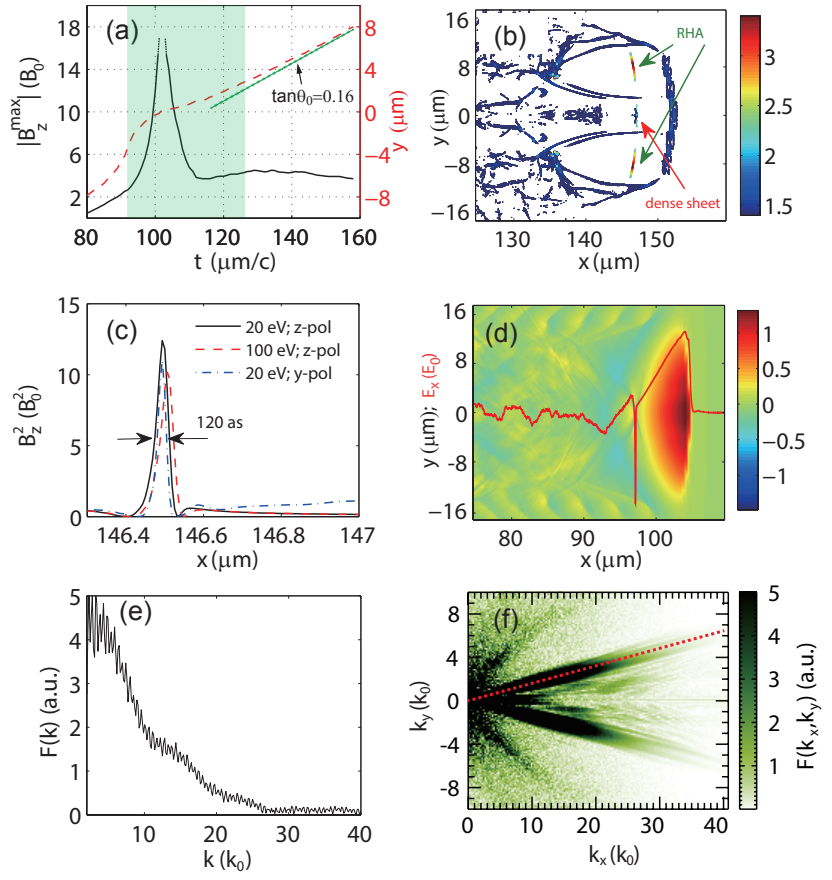

FIG. 2: (Color online). (a) Evolution of the maximal radiation field $\left|B_{z}^{\max }\right| / B_{0}$ (solid) and associated $y$ coordinate (dashed) of the RHA pulse versus time. The shaded region refers to beam crossing stage as explained in the text. The green dotted line indicates the slope of propagation direction. (b) Contour plots of the electron density $n / n_{c}$ and $\left|B_{z} / B_{0}\right|$ (sharing the same color bar) at $t=160 \mu \mathrm{m} / \mathrm{c}$. The wake wave has developed a complex broken-wave pattern, in particular to the sides bordering unperturbed plasma and downstream. The only features relevant to this paper are the tiny dense sheet of relativistic electrons (marked by red arrow) and the RHA pulse (marked by green arrows). (c) Line-outs of $\left(B_{z} / B_{0}\right)^{2}$ along $y=8.32 \mu \mathrm{m}$ at $t=160 \mu \mathrm{m} / \mathrm{c}$ for three cases corresponding to different initial plasma temperatures $(20$ and $100 \mathrm{eV}$ ) and $y$ or $z$ polarization of driving laser. (d) Snapshot of longitudinal electric field $E_{x}$ at $t=110 \mu \mathrm{m} / \mathrm{c}$; the red curve $(\times 10)$ shows on-axis profile. (f) $2 \mathrm{D}$ spatial spectrum of the RHA pulse at $t=160 \mu \mathrm{m} / \mathrm{c}$ and line-out along the dashed line is given in (e).

pulse evolves with time, where $B_{0}=m_{e} \omega_{0} / e$ is the normalizing field. Combining with Fig. 1(b), we see that the attosecond pulse emerges in three stages: field amplification during initial contraction, beam crossing on axis, and finally annular beam propagating along a cone. At the stage of beam crossing, the emission is focused to a maximum of $B_{z}^{\max } / B_{0} \geq$ 18, indicated by the shaded region in Fig. 2(a). This corresponds to a peak intensity greater than $7 \times 10^{20} \mathrm{~W} / \mathrm{cm}^{2}$, almost 10 times larger than the incident driving pulse. After beam crossing on axis (e.g., $t>130 \mu \mathrm{m} / \mathrm{c}$ ), the upper and lower branch of the pulse propagate separately in space and the actual peak field shows up as $B_{z}^{\max } / B_{0}=4.5$, corresponding to $4.3 \times 10^{19} \mathrm{~W} / \mathrm{cm}^{2}$ in intensity or $\sim 2 \times 10^{13} \mathrm{~V} / \mathrm{m}$ in field strength. It then slowly decays due to diffraction.

Figure 2(b) shows how the RHA pulse propagates at later time in the wake wave. It is found that the initial quasi-1D wake is evolving into a complex broken-wave pattern due to laser evolution [26]. The half-cycle pulse as short as 120 as 
[see Fig. 2(c)] is propagating inside the density bubble, but deviated from the center axis. The corresponding spatial spectrum of the pulse given in Fig. 2(f) shows clearly two branches, each with a small divergence. The line-out along one of the branches given in Fig. 2(e) shows a broad-band XUV spectrum extending up to $k / k_{0} \simeq 25$ or about $40 \mathrm{eV}$ in photon energy, where $k_{0}=2 \pi / \lambda_{0}$. In the present simulation case, the annular beam carries a total energy of $\sim 12 \mathrm{~mJ}$, which is about $2 \times 10^{-3}$ of the incident laser energy.

The RHA generation is found to be insensitive to finite thermal temperatures of the ambient plasma electrons. The emission profile hardly changes when increasing $T_{e}$ up to $100 \mathrm{eV}$ as depicted in Fig. 2(c). Also shown in this subfigure is the resulting emission profile when switching the polarization direction of the driving pulse from $z$ to $y$ (within the simulation plane) axis. Few differences are observed, indicating that the polarity of the attosecond emissions is independent of the driving laser, but only determined by the transverse contraction motion of the sheet electrons. We have confirmed this by using 3D PIC simulations (not shown here) that the annular attosecond light beam is uniform in intensity azimuthally, corresponding to radial polarization. Notice that full-scale 3D simulations for the present configuration generally cause extremely heavy computational loads due to the wide box dimensions and high resolutions required for both longitudinal and transverse directions, so that they have to be conducted with much lower resolutions compared with the above $2 \mathrm{D}$ runs. However, they confirm very well the half-cycle profile and the radial polarity of the attosecond emissions.

\section{Simplified model for attosecond pulse generation}

The basic dynamics of RHA generation can be derived from a simplified model. It assumes that a flat, monoenergetic electron sheet of delta-like density profile $n_{s}=\delta\left(x / \sigma_{s}\right)$ with finite areal density $\sigma_{s}=\int n_{s} d x$ and no initial transverse momentum is injected into a wakefield of uniform accelerating and focusing fields. Coherent radiation from the electron sheet is then described by the $1 \mathrm{D}$ wave equation $\left(\partial^{2} / \partial x^{2}-c^{-2} \partial^{2} / \partial t^{2}\right) E_{y, r}=\epsilon_{0}^{-1} c^{-2} \partial J_{y, r} / \partial t$, where $J_{y, r}=-e c \beta_{y, r} n_{s}$ is the radiating current with $\beta_{y, r}=$ $\int_{\tau_{0}}^{\tau_{1}} d \beta_{y} \ll 1$ the velocity integrated over a short transverse acceleration [10]. The radiated field $E_{y, r}$ can be first calculated in the rest frame of the sheet with Lorentz factor $\sim \gamma$ and then transformed back to the laboratory frame. The result is given by

$$
E_{y, r} \simeq\left(\frac{\sigma_{s}}{\epsilon_{0}}\right) \beta_{y, r}^{R} \gamma
$$

where $\beta_{y, r}^{R}$ denotes the velocity $\beta_{y, r}$ in the rest frame. Equation (2) indicates that the radiated power, $\propto\left|E_{y, r}\right|^{2} \propto \gamma^{2}$, grows with the electron energy. On the other hand, the Lorentz factor $\gamma$ evolves following from $d \gamma / d t=-\vec{\beta}_{x} \cdot \vec{E}_{x}-\vec{\beta}_{y} \cdot \vec{E}_{y}$. Taking into account the radiated fields $E_{y, r}$, the total fields can be expressed as $\vec{E}_{x}=\vec{E}_{x, w}$ and $\vec{E}_{y}=\vec{E}_{y, w}+\vec{E}_{y, r}$, where $\vec{E}_{x, w}$ and $\vec{E}_{y, w}$ are the longitudinal and transverse wake fields,
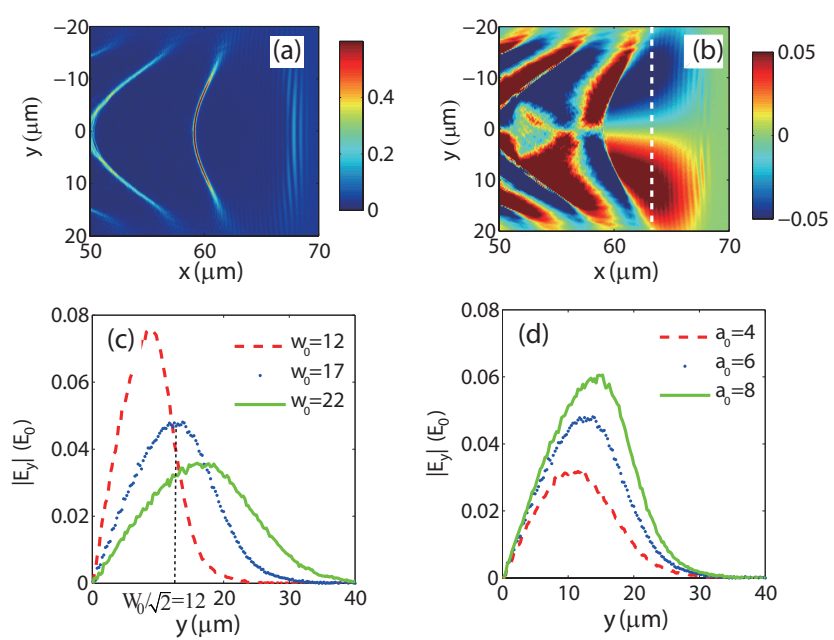

FIG. 3: (Color online). Snapshots of (a) electron density $n_{e} / n_{c}$ and (b) transverse wake field $E_{y, w} / E_{0}$ taken when the first wake wave period is approaching the density plateau. Line-outs of $E_{y, w}$ along $x=63 \mu \mathrm{m}$ [see the vertical dashed line in (b)] for (c) different laser waists and for (d) different laser amplitudes.

respectively. Inserting the above field components as well as the expression of Eq. (2), the energy equation reads

$$
\frac{d \gamma}{d t}+\frac{\sigma_{s}}{\epsilon_{0}} \beta_{y, r}^{R} \beta_{y} \gamma-\beta_{y} E_{y, w}-\beta_{x} E_{x, w}=0 .
$$

Assuming $\gamma(t=0)=1$ initially, wakefield acceleration first boosts $\gamma$ factors rapidly, which then saturates due to radiation damping at a rate $\left(\sigma_{s} / \epsilon_{0}\right) \beta_{y, r}^{R} \beta_{y}$. Saturation occurs for $\vec{\beta}_{x}$. $\vec{E}_{x}+\vec{\beta}_{y} \cdot \vec{E}_{y}=0$, which leads to the saturated field

$$
E_{y, r}^{s a t} \simeq E_{x, w} / \tan \theta+E_{y, w}
$$

where $\tan \theta=\beta_{y} / \beta_{x}$ is evaluated at the saturation instant. It is clear from the above analysis that wakefield acceleration has played a crucial role in RHA generation. At this point, the present case differs from the half-cycle pulse generation from the double-foil scheme, where the relativistic electron sheet emerging from the second foil is almost fixed in $\gamma$ and is supposed to radiate immediately the strongest fields [10]. Equation (4) further suggests that $E_{y, r}^{s a t}$ can be large provided that $\tan \theta \simeq \beta_{y}$ is small initially and, in an ideal case, the saturated level can also be maintained for a long period.

\section{Additional multi-dimensional effects}

There are a couple of effects not yet included in the above simplified model where a flat electron sheet is assumed. In the sample simulation, the injected sheet is not perfectly flat but slightly curved as seen in Fig. 1(a). Owing to the sharp longitudinal injection, a curved wake wavefront $[16,17]$ will directly map into the initial spatial profile of the injected electron sheet. More importantly, the injected electrons will also 
inherit certain transverse velocities. These will cause transverse contraction during propagation even without the focusing wake fields, and the radiative process is ultimately limited by deformation of the sheet profile.

To see qualitatively what the initial transverse velocity distribution is and how it scales with laser parameters, we analyze single electron dynamics in a wakefield. To the zeroth-order, the normalized transverse velocity changes as $d \beta_{y} / d t=(1 / \bar{\gamma})\left(-E_{y, w}+\beta_{x} B_{z, w}\right)$, where $B_{z, w}$ is the quasistatic magnetic fields generated in a laser wake [27], and $\bar{\gamma}$ is the lorentz factor averaged over laser cycles. The laser ponderomotive force is weak in transverse direction and not included. For the case of sharp injection, the initial velocities of sheet electrons are equal to that shortly before trapping. Considering a cold start for plasma electrons, the transverse velocity at the apex of the first wake wave crest is given by $\beta_{y}=-\int_{-\infty}^{t}(1 / \bar{\gamma}) E_{y, w} d t$, where the contribution from $B_{z, w}$ is omitted since $\beta_{x}$ changes sign during a plasma oscillation. As a consequence, the initial transverse velocities are mainly determined by the transverse wake fields $E_{y, w}$, so larger $E_{y, w}$ generally results in larger $\beta_{y}$.

The electron density of the wake wave when approaching the density plateau is plotted in Fig. 3(a) and the corresponding transverse wake fields $E_{y, w}$ in Fig. 3(b). The transverse line-outs of $E_{y, w}$ along $x=63 \mu \mathrm{m}$ are given in Figs. 3(c) and 3 (d) for different laser parameters. It is found that they share the similar profile with a maximum at $y=W_{0} / \sqrt{2}$. Notice that this sort of distribution obtained in the present highly nonlinear quasi-1D regime is similar to that of a linear wake, i.e., $E_{y, w} \propto y \exp \left(-y^{2} / W_{0}^{2}\right)$, if a Gaussian (transverse profile) driving laser is used [23]. Following the above qualitative analysis and considering that the radius of injected electron sheet normally satisfies $r_{s} \leq W_{0} / \sqrt{2}$ [19], the sheet electrons at larger radii will have larger initial transverse velocities. As we shall see, this velocity distribution is useful for the interpretation of a couple of simulation results beyond the above simplified model.

Below we adopt particle tracking to illustrate these additional effects. A group of electrons uniformly picked from the lower half of the sheet are tracked. As seen in Figs. 4(a-c), the electrons are injected at about $t=74 \mu \mathrm{m} / \mathrm{c}$ and focused to the axis at about $t=102 \mu \mathrm{m} / \mathrm{c}$. During this period, these electrons develop a broad $\gamma$ spread primarily due to longitudinal injection dynamics. Figure 5 shows the trapped electron sheet during the contraction phase. It is found that the sheet could be treated as a sequence of sub-layers, each of which is monochromatic in energy and can be well described by the simplified model shown above. As we have pointed out, the overcritical density and ultrathin feature of the sheet ensure the coherency of the overall attosecond emissions. Figure 4(d) further shows snapshots of the propagation angles $\theta \simeq\left(180^{\circ} / \pi\right) \arctan \beta_{y}$ of these electrons at different times, either shortly before injection or during the contraction phase. It is seen that the corresponding $\beta_{y}$ values are larger for larger radii and they further increase slowly with time due to transverse acceleration. This velocity distribution is in agreement with the above qualitative analysis.

The transverse velocity distribution suggests that the disk-
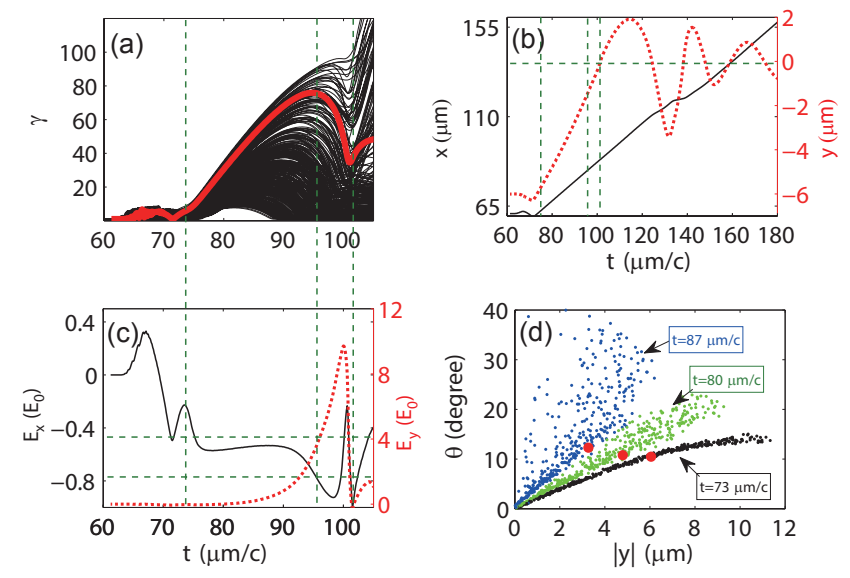

FIG. 4: (Color online). Particle tracking of 400 electrons uniformly picked from the lower half of the electron sheet at $t=80 \mu \mathrm{m} / \mathrm{c}$. (a) Evolution of $\gamma$ versus time. The red (thicker) curve highlights a typical electron which is initially located near the center of the lower half and belongs to the most energetic ones during acceleration. Time histories are presented for this electron in terms of (b) its coordinates $x$ (solid) and $y$ (dotted) and (c) electric fields $E_{x}$ (solid) and $E_{y}$ (dotted) felt by it. The vertical dashed lines (from left to right) mark, respectively, the time instant of injection, $\gamma$-saturation and reaching the center axis $y=0$. (d) Distributions of propagation angle $\theta \simeq$ $\left(180^{\circ} / \pi\right) \arctan \beta_{y}$ for these electrons versus radial coordinate $|y|$ at the times indicated; $t=73 \mu \mathrm{m} / \mathrm{c}$ corresponds to shortly before injection. The red (thicker) dots refer to the typical electron selected in (a).

like electron sheet can be further subdivided into a sequence of ring-shaped segments defined by their $\beta_{y}$ values. Applying the simplified model to each ring segment and rotating the coordinate frame by angle $\theta \simeq \arctan \beta_{y}$ with respect to the normal $x-y$ axis, the resulting pointing direction of the radiated electric fields will deviate somewhat from $y$ axis; see the schematic drawing in Fig. 1(b). An evidence for the angle deviation is given in Fig. 2(d), where a sharp negative $E_{x}$ field shows up at the on-axis overlapping joint during the stage of beam crossing. Moreover, the fields emitted from segments

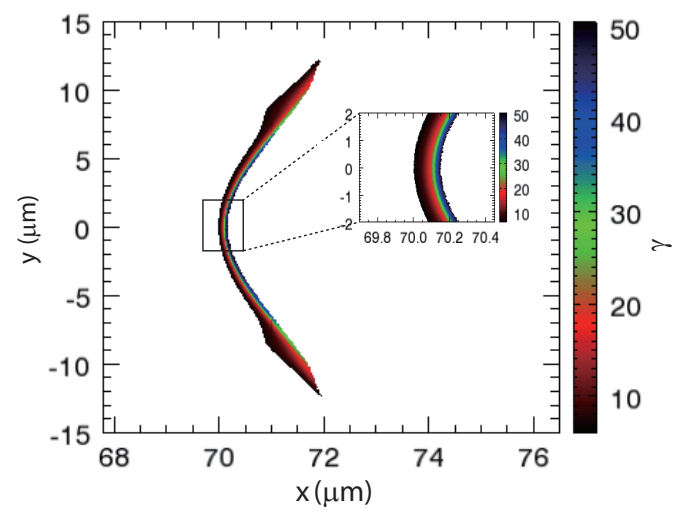

FIG. 5: (Color online). Snapshot of injected electron sheet at $t=$ $83 \mu \mathrm{m} / \mathrm{c}$ colored according to $\gamma$. The inset shows zoom-in plot of the small rectangle region. 

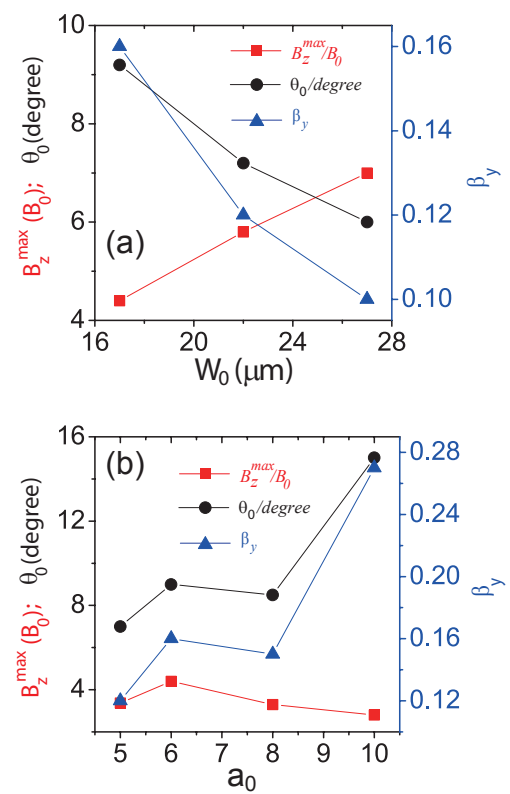

FIG. 6: (Color online). The maximal radiation field $B_{z}^{\max }$ (solid square), $\theta_{0}$ defined in Fig. 1 (solid circle), and $\beta_{y}$ for the ring segment (as explained in the text) emitting the maximal field (solid triangle) versus (a) driving laser waist $W_{0}$ while keeping $a_{0}=6$ and $n_{0}=0.04 n_{c}$ fixed, and versus (b) laser amplitude $a_{0}$ while keeping $W_{0}=17 \mu \mathrm{m}$ and $n_{0}=0.04 n_{c}$ fixed.

of different $\beta_{y}$ (or $\theta$ ) will converge due to propagation. This just accounts for the focusing effect observed in Fig. 2(a). The finally observed RHA pulse builds up from all segment contributions weighted according to Eq. (2). As it turns out, largest contributions stem from ring segments with medium radius. For the present case, the conical angle $\theta_{0}$ of the maximal radiation field is measured to be $\tan \theta_{0} \simeq 0.16$ as shown in Fig. 2(a). To evaluate this peak field, a typical electron is highlighted in Fig. 4; it is initially located near the center of the lower half and belongs to the most energetic ones after acceleration. According to Eq. (4), the peak field amounts to $E_{y, r}^{s a t} \simeq E_{x} / \tan \theta_{0}=4.7$, where $E_{x} \simeq 0.75$, extracted from Figs. 4(a) and 4(c), is the longitudinal electric field felt by the electron when its Lorentz factor $\gamma$ becomes saturated, and $E_{y, w} \ll E_{x, w}$ is omitted here. This result is in fair agreement with $B_{z}^{\max } / B_{0}=4.5$ observed after $t \sim 130 \mu \mathrm{m} / \mathrm{c}$.

We have further checked that the RHA generation is robust against changing laser and plasma parameters. Following Figs. 3(c) and the analysis therein, larger focal spots of the driving pulse lead to smaller initial transverse velocities of the injected electron sheet, thus higher field amplitudes are expected according to Eq. (4). This is verified in Fig. 6(a), where $B_{z}^{\max }$ and $\theta_{0}$ scale almost linearly with the focal spot $W_{0}$. While scanning the laser amplitude $a_{0}$ for fixed plasma density of $n_{0}=0.04 n_{c}$ and focal spot of $W_{0}=17 \mu \mathrm{m}$, an optimal value of $a_{0} \sim 6$ for RHA generation (means producing the most intense field) exists as seen in Fig. 6(b). This is because the sheet injection requires high enough driving laser amplitudes [19]; while for too strong drivers, the
quasi-1D wake excitation will be violated for fixed laser focal spot. The scaling dependence with the plasma density $n_{0}$ becomes even more complicated, because the laser conditions have to be adjusted accordingly to make the sheet injection possible.

\section{Summary and discussions}

In conclusion, we have identified a novel attosecond burst of coherent synchrotron-like radiation from laser wakefield acceleration. The attosecond feature does not derive from solid surface layers [9] or ultrathin solid foils [10], but arises from intrinsic features of nonlinear plasma waves, namely steepening and breaking. It makes use of quasi-1D wake wave breaking, allowing to trap a dense electron sheet into the wakefield. The injected electron sheet contracts radially by focusing wake fields, while boosted in energy primarily by longitudinal wakefield acceleration, and is found to emit an intense half-cycle attosecond pulse. Pulse energies over $10 \mathrm{~mJ}$ can be obtained with the laser-to-RHA conversion efficiency exceeding $10^{-3}$. Besides being a bright source for attosecond applications, this burst will also provide useful diagnostics for wavebreaking dynamics [28] in the highly nonlinear quasi-1D regime and corresponding electron sheet injection [19].

It is worthwhile to emphasize that the coherent half-cycle attosecond pulse emerges at the initial contraction phase of the electron sheet after injection. After completely focused to the axis, these electrons are confined within a smaller radius [see Fig. 1(a) and Fig. 2(b)] due to wake evolution and can transport for a long distance with highly relativistic energies. They perform significant betatron oscillations and shall also emit incoherent keV-level x-rays as those normally found with wakefield accelerated electrons [15]. Therefore, in real experiments, the present scheme will deliver both a coherent attosecond XUV pulse laterally and an incoherent X-ray beam in the forward direction. Since the number of trapped electrons in the quasi-1D regime with controlled injection can be made higher (such as nC) [19], the present configuration may also provide the possibility for brighter betatron radiations with better control.

The required experimental conditions for RHA generation are within current technical capabilities. Petawatt lasers, presently coming up, will provide sufficiently high power for the high intensities and relatively broad focal spots preferable here. The sample simulation case shown above used a single $350 \mathrm{TW}$ driving pulse delivering $6.5 \mathrm{~J}$ in $\sim 20 \mathrm{fs}$. These parameters are well within the ELI-facility capabilities [29] and are also becoming available commercially. The conical angle of the RHA beam is typically less than $10^{\circ}$, thereby allowing refocusing even at half-meter distance by a concentric mirroring tube with a diameter less than $16 \mathrm{~cm}$; such techniques have already been implemented in the measurement of wakefield-based electro-optic shocks [30]. The RHA pulse should also be easily distinguished from other sources due to its radial polarization and hollow pattern.

\section{Acknowledgments}


ZMS would like to thank the OSIRIS Consortium at UCLA and IST (Lisbon, Portugal) for providing access to OSIRIS 2.0 framework. FYL acknowledges helpful discussion with Prof. H.-C. Wu. MC appreciates support from National 1000 Youth Talent project of China. This work is supported in part by the National Basic Research Program of China (Grant No.
2013CBA01504), the National Natural Science Foundation of China (Grant No. 11121504, 11374210 and 11374209), and the MOST international collaboration project (Grant No. 2014DFG02330). Simulations were supported by Shanghai Supercomputer Center and by the center for high performance computing at SJTU.
[1] F. Krausz and M. Ivanov, Rev. Mod. Phys. 81, 163 (2009).

[2] P. B. Corkum and F. Krausz, Nat. Phys. 3, 381 (2007); K. Midorikawa, Jap. J. Appl. Phys. 50, 090001 (2011).

[3] U. Teubner and P. Gibbon, Rev. Mod. Phys. 81, 445 (2009).

[4] G. A. Mourou, T. Tajima, and S. V. Bulanov, Rev. Mod. Phys. 78, 309 (2006); G. Mourou and T. Tajima, Science 331, 41 (2011).

[5] A. Pukhov, T. Baeva, D. an der Brügge, and S. Münster, Eur. Phys. J. D 55, 407 (2009); G. D. Tsakiris, K. Eidmann, J. Meyer-ter-Vehn, and F. Krausz, New J. Phys. 8, 19 (2006).

[6] N. M. Naumova, J. A. Nees, I. V. Sokolov, B. Hou, and G. A. Mourou, Phys. Rev. Lett. 92, 063902 (2004).

[7] J. A. Wheeler, A. Borot, S. Monchocé, H. Vincenti, A. Ricci, A. Malvache, R. Lopez-Martens and F. Quéré, Nat. Photonics 6, 829 (2012); K. T. Kim, C. Zhang, T. Ruchon, J. Hergott, T. Auguste, D. M. Villeneuve, P. B. Corkum, and F. Quéré, Nat. Photonics 7, 651 (2013).

[8] V. V. Kulagin, V. A. Cherepenin, M. S. Hur, and H. Suk, Phys. Rev. Lett. 99, 124801 (2007); H. C. Wu, J. Meyer-ter-Vehn, J. Fernández, and B. M. Hegelich, Phys. Rev. Lett. 104, 234801 (2010).

[9] D. an der Brügge and A. Pukhov, Phys. Plasmas 17, 033110 (2010); B. Dromey, S. Rykovanov, M. Yeung, R. Hörlein, D. Jung, D. C. Gautier, T. Dzelzainis, D. Kiefer, S. Palaniyppan, R. Shah, J. Schreiber, H. Ruhl, J. C. Fernandez, C. L. S. Lewis, M. Zepf, and B. M. Hegelich, Nat. Physics 8, 804 (2012); M. Yeung, B. Dromey, S. Cousens, T. Dzelzainis, D. Kiefer, J. Schreiber, J. H. Bin, W. Ma, C. Kreuzer, J. Meyer-ter-Vehn, M. J. V. Streeter, P. S. Foster, S. Rykovanov, and M. Zepf, Phys. Rev. Lett. 112, 123902 (2014).

[10] H. C. Wu and J. Meyer-ter-Vehn, Nat. Photonics 6, 304 (2012).

[11] T. Tajima and J. M Dawson, Phys. Rev. Lett. 43, 267 (1979); E. Esarey, C. Schroeder, and W. Leemans, Rev. Mod. Phys. 81, 1229 (2009).

[12] I. Kostyukov, E. Nerush, A. Pukhov, and V. Seredov, Phys. Rev. Lett. 103, 175003 (2009).

[13] G. R. Plateau, C. G. R. Geddes, D. B. Thorn, M. Chen, C. Benedetti, E. Esarey, A. J. Gonsalves, N. H. Matlis, K. Nakamura, C. B. Schroeder, S. Shiraishi, T. Sokollik, J. van Tilborg, Cs. Toth, S. Trotsenko, T. S. Kim, M. Battaglia, Th. Stöhlker, and W. P. Leemans, Phys. Rev. Lett. 109, 064802 (2012).

[14] A. Pukhov and J. Meyer-ter-Vehn, Appl. Phys. B 74, 355 (2002); W. Lu, C. Huang, M. Zhou, W. B. Mori and T. Katsouleas, Phys. Rev. Lett. 96, 165002 (2006).

[15] A. Rousse, K. T. Phuoc, R. Shah, A. Pukhov, E. Lefebvre, V. Malka, S. Kiselev, F. Burgy, J. P. Rousseau, D. Umstadter, and D. Hulin, Phys. Rev. Lett. 93, 135005 (2004); S. Corde, K. Ta
Phuoc, G. Lambert, R. Fitour, V. Malka, and A. Rousse, Rev. Mod. Phys. 85, 1 (2013).

[16] N. H. Matlis, S. Reed, S. S. Bulanov, V. Chvykov, G. Kalintchenko, T. Matsuoka, P. Rousseau, V. Yanovsky, A. Maksimchuk, S. Kalmykov, G. Shvets and M. C. Downer, Nat. Physics 2, 749 (2006).

[17] S. V. Bulanov, T. Esirkepov and T. Tajima, Phys. Rev. Lett. 91 , 085001 (2003); M. Kando, Y. Fukuda, A. S. Pirozhkov, J. Ma, I. Daito, L. M. Chen, T. Z. Esirkepov, K. Ogura, T. Homma, Y. Hayashi, H. Kotaki, A. Sagisaka, M. Mori, J. K. Koga, H. Daido, S. V. Bulanov, T. Kimura, Y. Kato and T. Tajima, Phys. Rev. Lett. 99, 135001 (2007).

[18] An electron sheet may be externally injected into a broad wake wave driven by petawatt lasers. Possible sheet production methods were proposed, for example, in Refs. [8]

[19] F. Y. Li, Z. M. Sheng, Y. Liu, J. Meyer-ter-Vehn, W. B. Mori, W. Lu and J. Zhang, Phys. Rev. Lett. 110, 135002 (2013).

[20] S. Corde, C. Thaury, A. Lifschitz, G. Lambert, K. Ta Phuoc, X. Davoine, R. Lehe, D. Douillet, A. Rousse and V. Malka, Nat. Commun. 4:1501 doi: 10.1038/ncomms2528 (2013).

[21] J. Mu, F. Y. Li, M. Zeng, M. Chen, Z. M. Sheng and J. Zhang, Appl. Phys. Lett. 103, 261114 (2013).

[22] R. Fonseca et al., Lecture Notes in Computer Science. 2331, 342-351 (Springer Berlin, Heidelberg, 2002).

[23] E. Esarey, B. A. Shadwick, P. Catravas, and W. P. Leemans, Phys. Rev. E. 65, 056505 (2002).

[24] T. Shiozawa, Classical Relativistic Electrodynamics, SpringerVerlag (Berlin, Heidelberg, 2004), pp77-82.

[25] K. Kan, J. Yang, A. Ogata, S. Sakakihara, T. Kondoh, K. Norizawa, I. Nozawa, T. Toigawa, Y. Yoshida, H. Kitahara, K. Takano, M. Hangyo, R. Kuroda and H. Toyokawa, Appl. Phys. Lett. 102, 221118 (2013).

[26] J. Xu, B. Shen, X. Zhang, M. Wen, L. Ji, W. Wang, Y. Yu and K. Nakajima, New J. Phys. 12, 023037 (2010).

[27] Z. M. Sheng, J. Meyer-ter-Vehn, and A. Pukhov, Phys. Plasmas 5, 3764 (1998).

[28] A. G. R. Thomas, S. P. D. Mangles, Z. Najmudin, M. C. Kaluza, C. D. Murphy, and K. Krushelnick, Phys. Rev. Lett. 98, 054802 (2007).

[29] ELI-Extreme Light Infrastructure Science and Technology with Ultra-Intense Lasers WHITEBOOK, edited by G. A. Mourou, G. Korn, W. Sandner, and J. L. Collier (THOSS Media GmbH, Berlin, 2011).

[30] M. H. Helle, D. Kaganovich, D. F. Gordon, and A. Ting, Phys. Rev. Lett. 105, 105001 (2010). 